\title{
How to Reduce Cerebral Plasy?
}

\section{Kazuo Maeda*}

Department of Obstetrics and Gynecology (Emeritus), Tottori University Medical School, Yonago, Japan

The cerebral palsy (CP) is infantile motility dysfunction caused by congenital or aquired brain damagrs. Its symptoms are paraplegia, quadriplegia, hemiplegia, spastic plegia, athetosis, ataxia and chorea without intelligence damage. Although maternal and perinatal infants' mortality were dramatically reduced, world CP production rate has not been reduced even after introduction of intense ante- and intrapartum fetal monitoring, despite domestic results of fetal heart rate monitoring reported the reduction of CP in Japan $[1,2]$. Some proposals probably to reduce infantile $\mathrm{CP}$ will be discussed in this editorial.

\section{Rapid Delivery in Cases in the Loss of Fetal Heart Rate Long Term Variability}

There will be a recommendation on the rapid delivery performed in the loss of fetal heart rate (FHR) long term variability in the nonreassuring fetal state (NRFS). The FHR variability will develop by the reaction of fetal brain to minor fetal movements in the analysis of actocardiogram, which recorded FHR and fetal movements, where the loss of FHR variability will be caused by hyoxic fetal brain damages causing CP in infants $[3,4]$. Therefore, the C-section after the loss of variability will prevent fetal death, however, fetal brain damage will not be prevented. Therefore, the author recommends to perform the rapid delivery including C-section before the loss of variability. Snce a severe loss of variability caused by the brain damage as svere as anencephaly may develop at least one in 10,000 deliveries, therefore, new management will reduce $100 \mathrm{CP}$ cases in $1,000.000$ births in a year in Japan, which will be $5 \%$ or more of CP cases developed in Japan in a year, if the CP development rate is $0.2 \%(2 / 1,000)$ of births. The assumption may be adopted to the world deliveries to reduce the $\mathrm{CP}$. The strategy to deliver the fetus before the loss of variability will be the artificial births in cases of the loss of acceleration, which precedes the loss of variability, in the decreased long term variability, severe FHR changes but preserving variability, or in high hypoxia index, which may be tentatively 20-24 points, while lower than the index in cases of the loss of variability [3,4], Hypoxia index is ( the sum of fetal bradycardia duration [min] x 100)/ the lowest FHR in the bradycardia lower than $110 \mathrm{pm}$. Further studies are required in the hypoxia index.

\section{Management of Preterm Neonatets having Paraventricular Echo Density (PVE)}

The $18 \%$ of preterm neonates, who maintained PVE until preterm birth, which was white periventricular B-mode image with high graylevel histogram width (GLHW) value, developed neonatal PVL and CP, which was $0.2 \%$ or less of total birth, while vanished PVE before the preterm birth or full term birthths did not develop neonatal PVL and CP [5]. Therefore, the strategies will (1) Pharmacological tocolysis of preterm labor untill full term births. Or (2) Sedation of preterm labor until full term birth by anesthesia or paralysis of uterus-brain nerve, which is the part of positive feed-back loop to produce regular preterm labor contraction [6]. Or (3) Ultrasonic study of preterm infant's brain immediately after preterm births to detect the PVE before developing PVL and the PVE will be treated until its disappearance by treatments with growth factor, hydrocortisone, erythropoietin etc, while their ffect has not been established.
Since neonatal hypoxic ischemic encephalopathy (HIE) causes neonatal brain damage, its treatment is important to prevent neonatal brain damage and CP. Hypothermic neonatal brain is the most effective treatment of HIE at present. Free radical scavenger or anti-glutamate has been tried in the HIE treatment. Since neonatal respiratory distress syndrome (RDS) may cause furher neonatal brain damage due to hypoxia, the RDS prediction in antenatal stage may support neonatal surfactant therapy after the birth.Non-invasive ultrasonic tissue characterization detected fetal lung immaurity, and $96 \%$ of neonatal RDS is predicted by the GLHW ratio of fetal lung and liver multiplied by gestational weeks. Fetal lung is immature if the parameter is less than 29 [7]. The technique may assess the effect of steroid therapy by repeated studies, because the it is fully non-invasive if compared to the fetal lung immaturity diagnosis with amniocentesis.Umbilical cord blood stem cell therapy was reported to be favorable to neurological damage [8]. Further improved pure stem cell therapy will be applied to the CP patient in the future.

\section{References}

1. Takeshita K, Ando Y, Ohtani K, Takashima S (1989) Cerebral palsy in Tottori, Japan. Benefits and risks of progress in perinatal medicine. Neuroepidemiology 8: 184-92

2. Tsuzaki T, Sekijima A, Morishita K, Takeuchi Y, Maeda K, et al. (1990) The survey on the perinatal variables and the incidence of cerebral palsy for 12 years before and after the application of the fetal monitoring system. Acta Obstet Ginecol jpn 42: 99-105.

3. Maeda K (2013) CP Prevention by early delivery before fetal brain damage in the loss of FHR variability, even in non-hypoxia. J Health Med Informat 4: 137.

4. Maeda K (2014) Modalities of fetal evaluation to detect fetal compromise prior to the development of significant neurological damage. J Obstet Gynecol Res 40: 2089-2094.

5. Yamamoto N, Utsu M, Serizawa M, Ohki S, Maeda K, et al. (2000) Neonata periventricular leukomalacia preceded by fetal periventricular echo density. Fetal Diag Ther 15: 198-208.

6. Maeda K (2013) Uterine contractions in normal labor developed by a positive feed back and oscillation. J Health Med Informat 4: 130.

7. Serizawa M, Maeda K (2010) Noninvasive fetal lung maturity prediction based on ultrasonic gray level histogram width. Ultrasound Med Biol 36: 1998-2003.

8. Wang S, Cheng H, Dai G, Wang X, Hua R, et al. (2013) Umbilical cord mesenchymal stem cell transplantation significantly improves neurological function in patients with sequelae of traumatic brain injury. Brain Res 1532 $76-84$

${ }^{*}$ Corresponding author: Kazuo Maeda, Department of Obstetrics and Gynecology (Emeritus), Tottori University Medical School, Yonago, Japan, Tel: 81-859-22-6856; E-mail: maedak@mocha.ocn.ne.jp

Received December 08, 2014; Accepted December 10, 2014; Published December 15, 2014

Citation: Maeda K (2015) How to Reduce Cerebral Plasy? J Health Med Informat 6: e134. doi:10.4172/2157-7420.1000e134

Copyright: (c) 2015 Maeda K. This is an open-access article distributed under the terms of the Creative Commons Attribution License, which permits unrestricted use, distribution, and reproduction in any medium, provided the original author and source are credited. 